\title{
Hard Decision-Based PWM for MIMO-OFDM Radar
}

\author{
Omar Daoud \\ Communications and Electronics Engineering Department, Philadelphia University, Amman, Jordan \\ Email: odaoud@philadelphia.edu.jo
}

Received 15 January 2015; accepted 6 February 2015; published 9 February 2015

Copyright (C) 2015 by author and Scientific Research Publishing Inc.

This work is licensed under the Creative Commons Attribution International License (CC BY). http://creativecommons.org/licenses/by/4.0/

c) (i) Open Access

\begin{abstract}
For the purpose of target localization, Multiple Input Multiple Output-Orthogonal Frequency Division Multiplexing (MIMO-OFDM) radar has been proposed. OFDM technique has been adopted in order to a simultaneous transmission and reception of a set of multiple narrowband orthogonal signals at orthogonal frequencies. Although multi-carrier systems such as OFDM support high data rate applications, they do not only require linear amplification but also they complicate the power amplifiers design and increase power consumption. This is because of high peak-to-average power ratio (PAPR). In this work, a new proposition has been made based on the Pulse Width Modulation (PWM) to enhance the MIMO-OFDM radar systems' performance. In order to check the proposed systems performance and its validity, a numerical analysis and a MATLAB simulation have been conducted. Nevertheless of the system characteristics and under same bandwidth occupancy and system's specifications, the simulation results show that this work can reduce the PAPR values clearly and show capable results over the ones in the literature.
\end{abstract}

\section{Keywords}

Multiple Input Multiple Output, Orthogonal Frequency Division Multiplexing, RADAR, Peak to Average Power Ratio, Pulse Width Modulation

\section{Introduction}

Many researchers have turned their attentions toward the Orthogonal Frequency Division Multiplexing (OFDM) scheme in order to provide high data rate applications under maintaining the spectral efficiency. Therefore, its clearly deployed in many broadband communication systems and protocols such as WiFi, WiMax, 4G and advanced LTE, Bluetooth-2. However, due to a high Peak-to-Average Power Ratio (PAPR), linear amplifiers suffer from low power efficiency under the utilization with multicarrier systems [1]-[5]. As a consequence, the cost of such devices; power amplifiers, mixers and analogue to digital convertor will be increased [1] [6]. OFDM 
signals are easily generated and produced by applying the Fast Fourier Transform (FFT) processing block and its Inverse (IFFT) [7]. This is due to its high speed processing in performing the needed operations, such as the transformation process, filtering and correlation [8]. On the other hand, Multiple Input Multiple Output (MIMO) concept has been found in the literature to enhance either the transmission capacity or the link robustness (independent or dependent information streams are transmitted via parallel sub-channels simultaneously). In contrast, in MIMO radar there is no need to construct parallel subchannels which are fully dependent on a multipath environment. This is due to that all transmitted information is known at the receiver side. Thus, the channel matrix is used only for the purpose of sensing the environment, as an example to determine the number of the targets, their locations and velocities [9]-[12].

In order to enhance the MIMO radar, which is adopting the OFDM technique, a new work has been proposed in this paper based on hard decision-based PWM technique to tackle one of the main deficiencies found in OFDM; namely PAPR.

This deficiency appears due to the addition process with different frequencies and phases of numerous waves, which leads to the need of high dynamic ranges transmitters. The predicted PAPR values in OFDM signal can be formed as [7]:

$$
\operatorname{PAPR}=\frac{\max _{0 \leq t \leq T}\left(|\mathbb{Z}(t)|^{2}\right)}{\left(\frac{1}{T} \int_{0}^{T}\left(|\mathbb{Z}(t)|^{2}\right) \mathrm{d} t\right)}
$$

Here, $\mathbb{Z}(t)$ is the transmitted OFDM symbol and could be found as $\mathbb{Z}(t)=\left(\sqrt{\frac{\left(\sum_{k=0}^{N-1}\left(X_{k} \cdot \mathrm{e}^{\left(j 2 \pi f_{k} t\right)}\right)\right)^{2}}{N}}\right)$,

which results from the modulation process of an $N$ symbols data block; $X_{k}$ with $f_{k}$, which are a set of orthogonal subcarriers for $k=0, \cdots, N-1$. The duration of OFDM symbol is denoted by $T$, which used to maintain the orthogonality for all values of $\mathrm{t}$ less than or equal to $T$. Moreover, to maintain the total transmitted power, the term $(1 / \sqrt{N})$ has been imposed.

Such deficiency causes transmission amplification and other circuitry limitations. Therefore, to overcome this problem, average signal power must be kept low to allow the transmission process of the higher average power to be in a fixed level. Then an improvement of the reception process will be attained based on improving the signal to noise ratio.

There are several propositions and techniques that either tackle the PAPR effects or address the linearity and power efficiency issues, such as filtering and clipping techniques; coding based techniques; artificial intelligence based techniques; and signal representation techniques as the envelope elimination and restoration techniques and the phase shifted sequence ones. This is in order to optimize a solution at the expense of several challenges, such as the degradation of the Bit Error Rate (BER); the decrement of the spectral efficiency due to the side information (SI) transmission; and the computational complexity [13]-[16].

This paper addresses the proposition of a new technique based on using the pulse width modulation (PWM) to overcome the PAPR problem effect. Consequently, the overall performance will be enhanced for the MIMO radar, which adopting the OFDM technique. As a result of considering the use of PWM, a basis of controlling the power electronics [17], an optimum solution will be provided to optimize the vital parameters of the existing work such as the speed, and the area. Moreover, the proposed work has been compared with either conventional OFDM systems in the literature or our previous published work in order to show the performance improvements before applying it to a MIMO-OFDM radar system.

PWM signal is easily generated by comparing the reference signal with a carrier one. Mainly, the input signal is used to determine the width of the generated PWM signal. This is clearly shown in the following mathematical representation

$$
\operatorname{PWM}(t)=\operatorname{sgn}(r(t)-c(t))
$$

where the generated PWM signal depends on the sign function of the subtraction process between the compared 
reference signal; $r(t)$ and the carrier signal; $c(t)$.

As basic PWM signal generation, there are two methods that help in producing the variable pulses widths; direct digital generation and uniformly sampled PWM. They can be distinguished by the focusing on the controlling criteria. In this paper, the second technique will be chosen, where a triangle clock signal is used to generate the uniformly sampled PWM signal. This is due to that it does not need high frequency clock signal. Moreover, the triangle clock signal is chosen over the other two types, Sawtooth or the inverted Sawtooth, due to that it has low number of dominant higher harmonics. The achieved benefit here concluded in reducing the needed system bandwidth [17] [18].

The rest of this work is introduced as follows: Section 2 describes the model of MIMO-OFDM radar signals based on PWM along with the analytical formulation in addition to the computational complexity. Section 3 presents simulation results and hardware implementations; finally, the conclusion is represented in Section 4.

\section{MIM0-OFDM Radar Signal Model-Based PWM}

\subsection{MIMO-OFDM Radar Systems Structure}

In [1], OFDM technique has the advantage of combating the frequency selective fading drawback for a narrowband system. This turns the researchers toward making use of such advantage to be imposed in MIMO radar systems. Therefore, the radar system performance will be improved by performing the target localization separately. This will be attained by making use of the combination of different orthogonal and narrowband sub-signals. As a result, the frequency selective fading deficiency is overcome by the frequency diversity utilization. Moreover, the complexity of MIMO-OFDM radar transmitters will be maintained at low level, since the used sub-bad waveforms designed to have same characteristics as the narrowband MIMO radar ones.

The baseband MIMO-OFDM transmitted matrix is defined in (1) by $\mathbb{Z}(t)$, where an $N$ sets of orthonormal signals have been sent simultaneously. The digital-to-analogue convertor (DAC) has been used to efficiently generate each OFDM symbol from $\mathbb{Z}(t)$ after the IFFT stage. For practical implementation using the IFFT, $\mathbb{Z}(t)$ should be oversampled [1] as shown below:

$$
\left[\mathbb{Z}_{i, j}(1) \cdots \mathbb{Z}_{i, j}(L)\right]=\operatorname{IFFT}\left(\left[\boldsymbol{X}_{i, j}(0) \cdots \boldsymbol{X}_{i, j}(k-1) \mathbf{0}_{1 \times(L-k)}\right]\right)
$$

Here, the IFFT of $\mathbb{Z}(t)$ matrix of the size of $(i \times j)$ will have an $L$ samples and defining its $l$-th sampling by $\mathbb{Z}_{i, j}(l)$, the element of $i$-th row and $j$-th column of the matrix $\boldsymbol{X}(k)$ is given by $\boldsymbol{X}_{i, j}(k)$, and $\mathbf{0}_{1 \times(L-k)}$ for zero padding when $L \geq k$. Accordingly, the produced $i$-th OFDM symbol will be processed and transmitted from the $i$-th antenna.

The next step after generating the OFDM baseband waveforms is the imposing of a guard interval process at the beginning of each OFDM symbol. This is attained by attaching a copy of the later OFDM symbol part at the beginning; namely a cyclic prefix process, which is introduced in order to maintain the orthogonality between the used sub-bands by MIMO operation and will be accomplished by making use of windowing techniques. Moreover, the MIMO-OFDM radar will make use of it in order to compensate for the shifts in time. This is clearly shown under the case of multiple targets at different ranges, where it guarantees the existence of the needed phase delay information inside the used window. This is true under a predefined separation range, which is based on the antenna array dimension and the used lengths for the transmitted symbol period and the cyclic prefix length. Therefore, the final transmitted baseband matrix is given as

$$
\mathbb{Q}(t)=\mathbb{Z}\left(t-T_{p}\right) W\left(\frac{t-T_{p}}{T_{s}}\right)+\mathbb{Z}\left(t+T_{s}-T_{p}\right) W\left(\frac{t}{T_{p}}\right)
$$

Here, $T_{p}$ is the length of the cyclic prefix, $T_{s}$ is the $\mathbb{Z}(t)$ elements period and equals $1 / f_{k}, W$ is a window function that has a value of unity when $t \in[0,1]$.

Moreover, in order to maintain the orthogonality condition, the antenna elements displacement should satisfy curtain threshold. In this work, to detect and estimate a target within $180^{\circ}$, the displacement; D should satisfy

$$
D \leq\left(c \times\left(T_{p}-T_{\text {target }}\right)\right)
$$

where, $c$ is the speed of light and $T_{\text {target }}$ is the target impulse response length. 
The imposing process of the cyclic prefix is clearly described in Figure 1, where a simultaneous transmission of three OFDM symbols from three antennas is accomplished.

In this work, and after the imposing of the guard interval; i.e. the cyclic prefix, a new processing block has been inserted to analyze the PAPR performance. This is to free the channel from the inter symbol interference (ISI) drawback. Moreover, this choice will reduce the hardware area under the consideration of hardware implementation.

\subsection{Proposition of PWM}

Returning to the implementation of the baseband OFDM signal; i.e. $\mathbb{Z}(t)$; in (1), and the possibility of producing high PAPR values. The overall system performance will be enhanced if the PAPR effect is efficiently reduced. This work focusing on proposing PWM based work that will produce constant amplitude signals by making use of the consecutive samples slope. It's considered as a novel technique that is attaining the maximum power amplifier efficiency issues, which will permit the ability of using nonlinear devices easily. Complementary cumulative distribution function (CCDF) curves give the statistical characteristics of the PAPR distribution in OFDM systems [19] [20]. CCDF curves show the probability of exceeding the PAPR a certain threshold for different signal to noise ratio values, i.e. the maximum power amplifier efficiency will be attained at the minimum CCDF value.

The proposed PWM work starts with reshaping the signal, $\mathbb{Z}(t)$, in (1) as blocks, each with length equals to the IFFT points as follows

$$
\mathbb{Z}(n, m)=\left(\sum_{k=0}^{N(m)-1}[\boldsymbol{X}(k, m)]\left[\boldsymbol{e}^{\left(j \frac{2 \pi k n}{N(m)}\right)}\right]\right) /(\sqrt{N(m)})
$$

Here, $m$ stands for the block index as defined in (7), and $N(m)$ denotes the block length.

$$
m=\left\{\begin{array}{rcrl}
0, & n & <N(0) \\
\rho, & \sum_{i=0}^{\rho-1} N(i) & \leq n<\sum_{i=0}^{\rho} N(i), \rho \geq 1
\end{array}\right.
$$

The reshaped result from (6) has been used to be processed in the production of a constant amplitude signal based on the PWM technique. This is clearly depicted and shown in Figure 2.

As shown in Figure 2, the conversion process is divided into the following stages:

\section{First stage:}

- In order to distinguish each symbol after the conversion process an extra zero sample has been added at the beginning of each OFDM symbol.

- The sampling rate has been increased in order to enhance the arcuracy of the conversion process; $\mathbb{Z}(n, m)$ will have extra samples under the use of new sampling rate $\grave{N}(m)$. This is clearly shown in (8) where $\mathbb{Z}(n, m)$ has been converted into a three vectors-matrix.

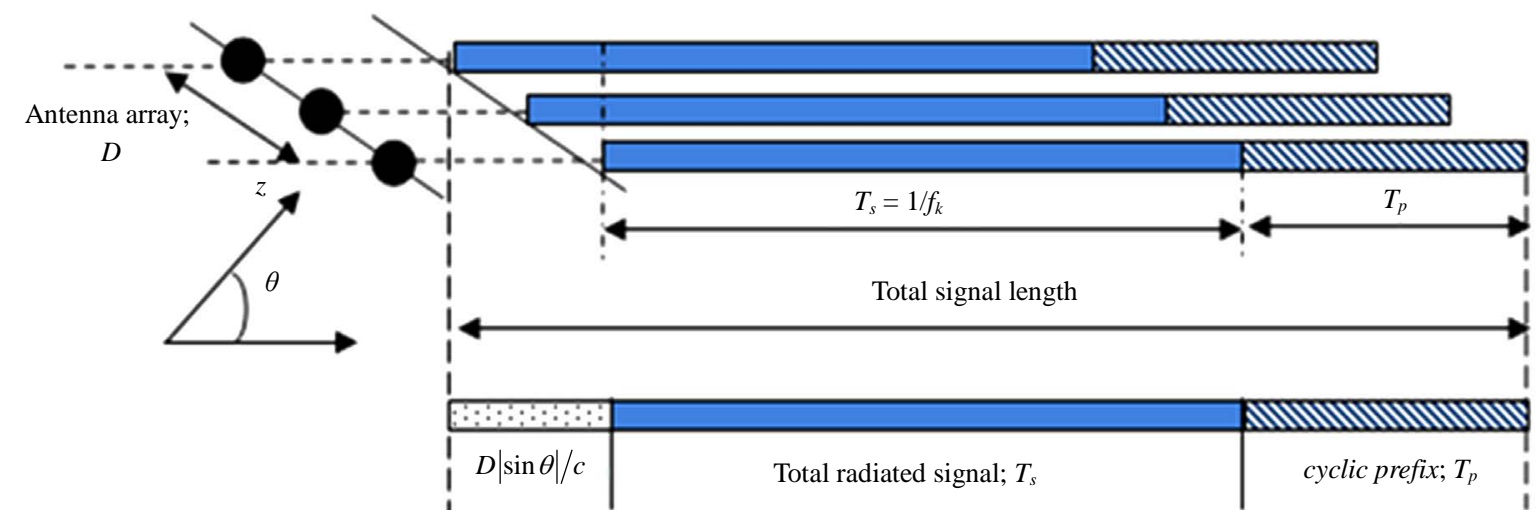

Figure 1. 3-MIMO-OFDM symbols transmission process. 


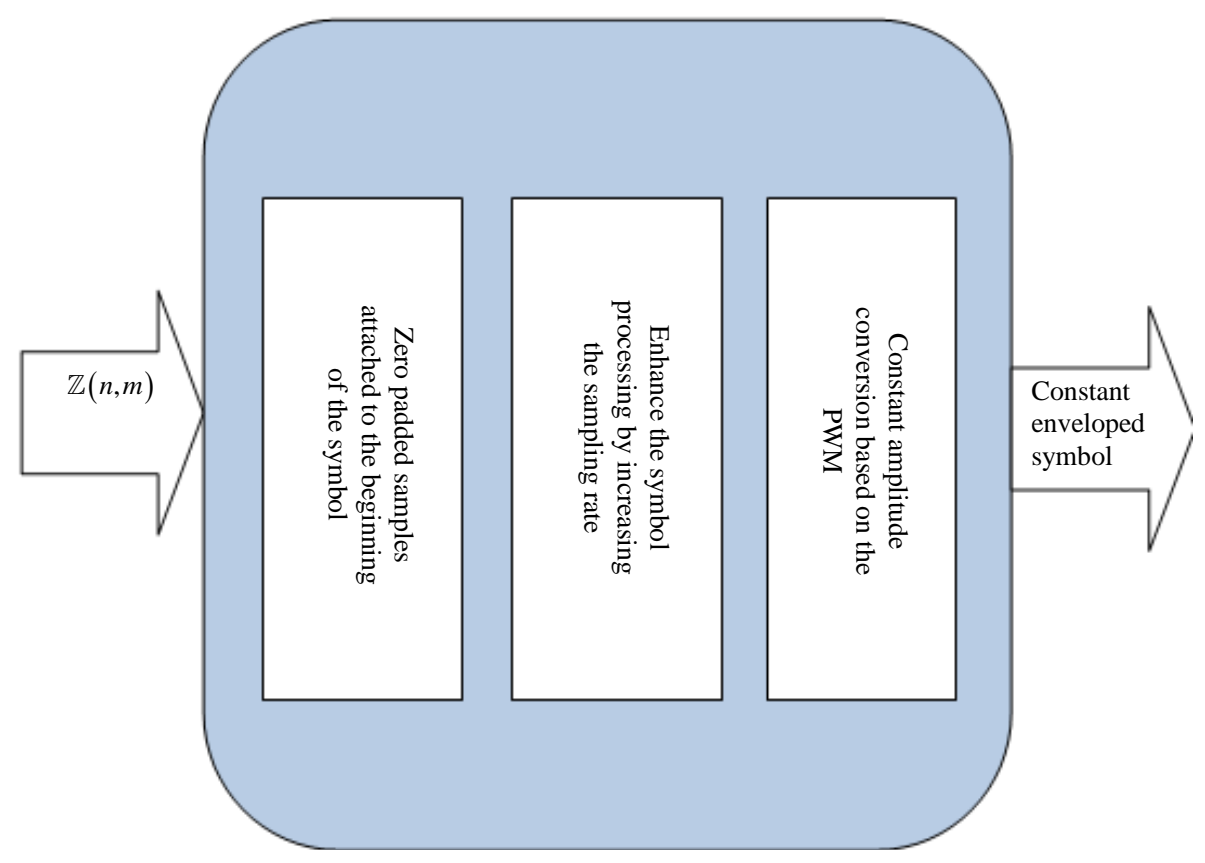

Figure 2. The conversion procedure.

$$
\mathbb{Z}(3, i)=\left\{\begin{array}{l}
\mathbb{Z}(n, m) \\
\mathbb{Z}(n, m)-\frac{1}{1+\grave{N}(m)}, \\
\mathbb{Z}(n, m)+\frac{1}{1+\grave{N}(m)}
\end{array}\right.
$$

where $i$ stands for the sample value; $i \in[1, \grave{N}(m)+N(m)]$.

\section{Second Stage:}

In this stage, the oversampled version of the OFDM symbol will be processed in order to produce a constant envelope version. For simplicity, the slope between two consecutive samples has been chosen as a comparison criterion. This criterion is depicted as follows in (9).

$$
\mathbb{Z}(i)= \begin{cases}\mathbb{Z}(i)=\mathbb{Z}(1,(i-1)), & \text { then } \mathbb{Z}(i)=0 \\ \mathbb{Z}(i)<\mathbb{Z}(2,(i-1)), & \text { then } \mathbb{Z}(i)=\text {-slope } \\ \mathbb{Z}(i)>\mathbb{Z}(3,(i-1)), & \text { then } \mathbb{Z}(i)=\text { +slope }\end{cases}
$$

These two stages are clearly described in Figure 3. It's divided into two main parts denoting the stages consequently.

As described earlier, Figure 3(a) depicts the process of the first stage and Figure 3(b) represents the procedure of the second stage. In Figure 3(b), the pre-process is divided into two main parts; the one that is responsible for simplifying the distinguishing process by adding a pre-known sample(s) at the beginning of the conventional symbol. In this work, the zero sample will do the expected results in either the transmission part or the reception one.

This is clearly found in Figure 4, where a zero sample has been attached at the beginning of the reshaped signal, i.e. $\mathbb{Z}(n, m)$. The second part deals with sampling rate of the modified $\mathbb{Z}(n, m)$; here extra samples have been imposed between the two consecutive samples by enhancing the sampling rate to be $\grave{N}(\mathrm{~m})$. Figure 5, shows the enhancement sampling rate for the signal with 108 samples instead of 12 samples. 


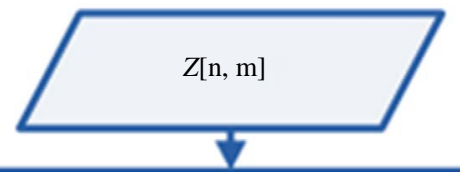

Imposing a distinguishing zero sample at the beginning of each symbol that will help in the recovery process

nhancing the conversion process by oversampling the modified symbol

Use shift window with size of the spacing between the samples of the original OFDM symbol

End for the first stage

(a)

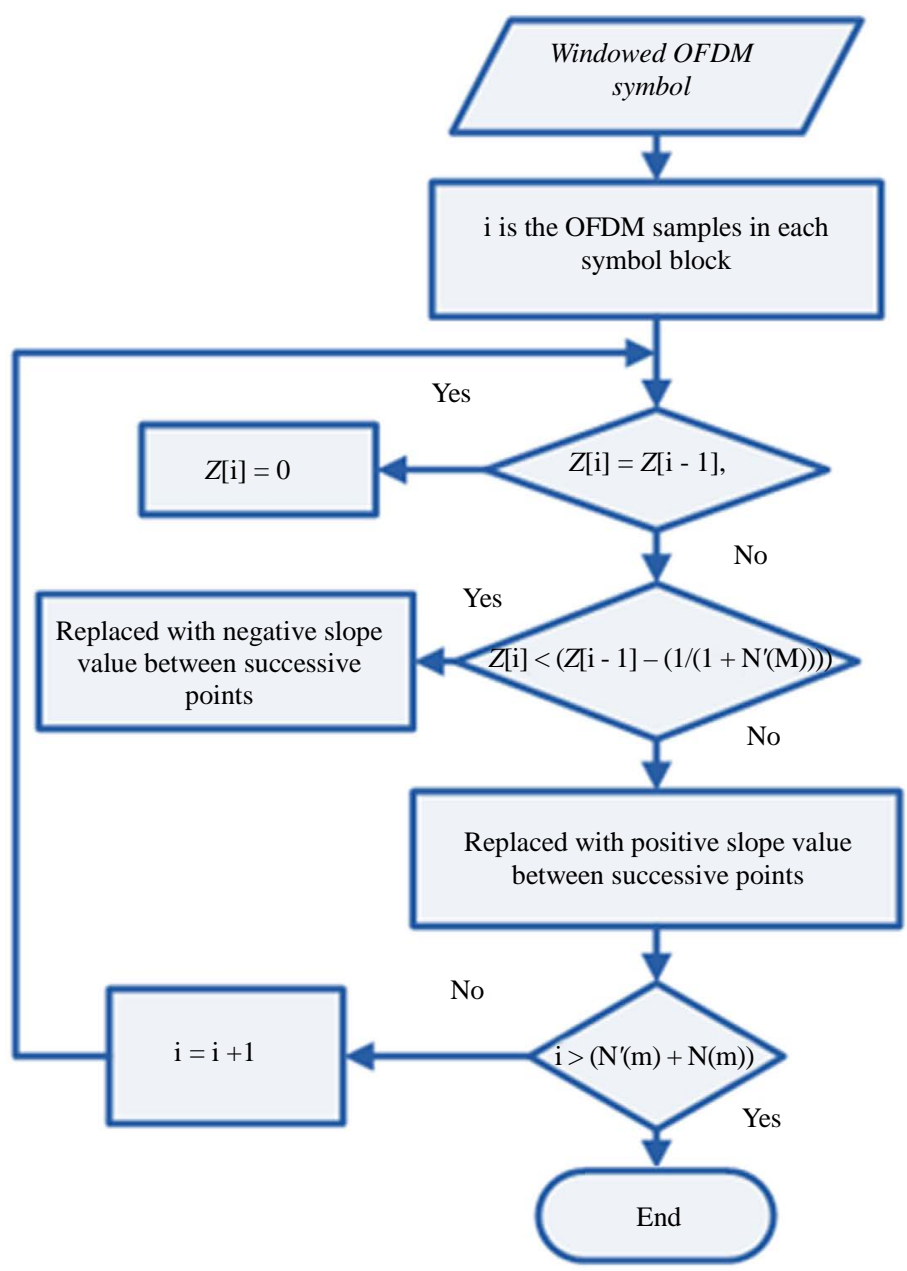

(b)

Figure 3. The proposed flowchart (a) First stage description; (b) Second stage description. 


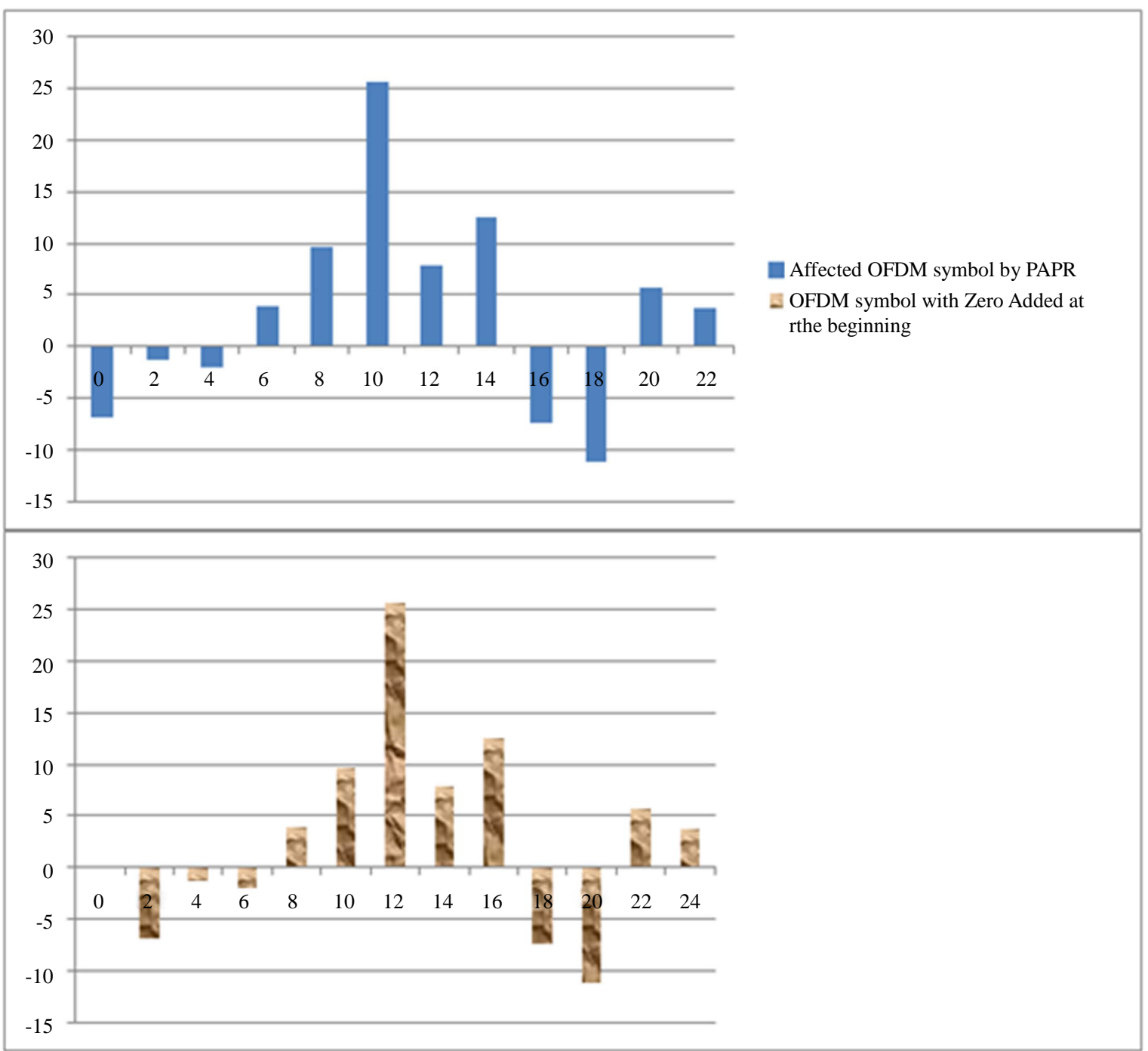

Figure 4. The process of adding the distinguishing zero sample.

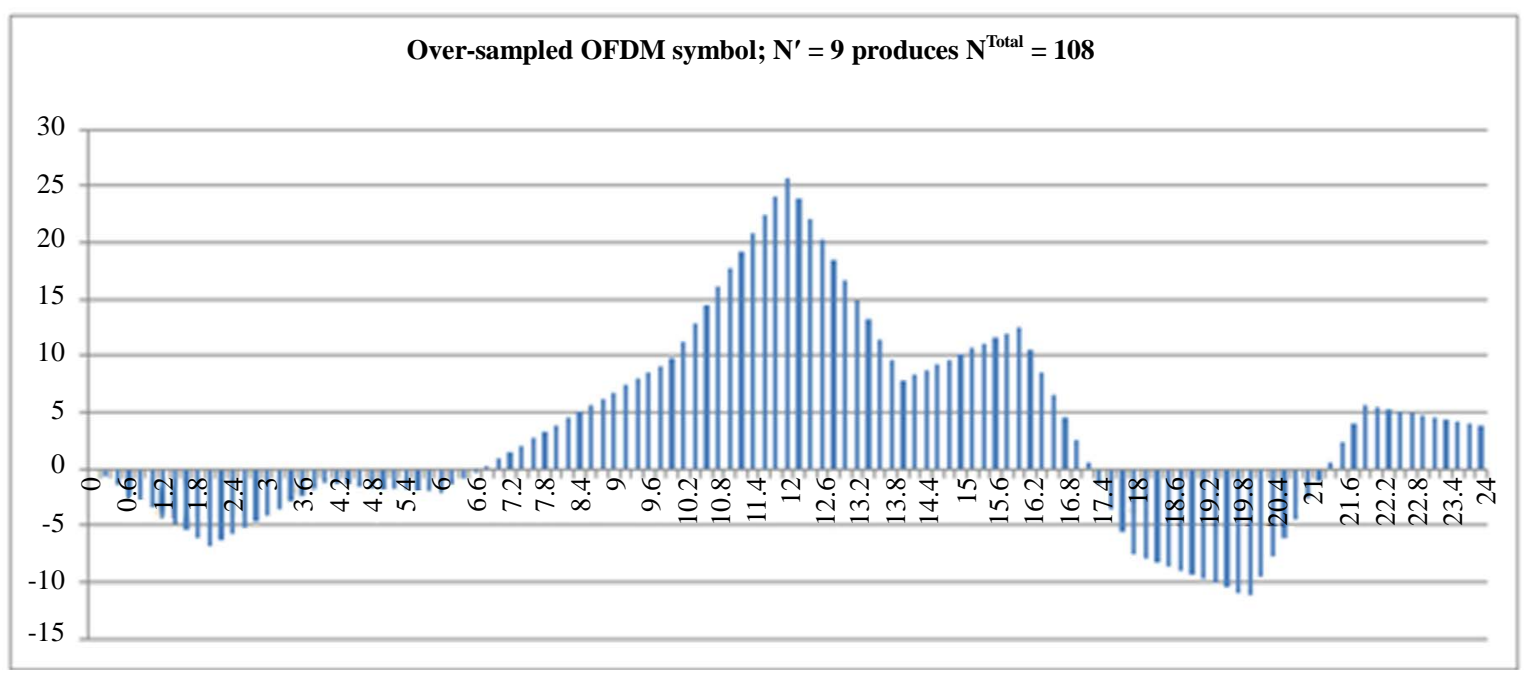

Figure 5. The over-sampled OFDM symbol. 
Figure 6 depicts the output results that are achieved from the second stage in the proposed work and shown in Figure 3(b), which is drawn from the proposed comparison formula in (9). In this figure, the idea of the proposed work has been showed up; i.e. the OFDM symbol has been converted to a constant enveloped symbol based on the slope between the adjacent samples.

The variation has been reduced and the peaks values have been diminished, consequently the PAPR values will be reduced. In addition, to simplify the proposed work, the hermitian structure of the OFDM systems could be exploited [21]. At this stage, the OFDM symbol is ready to be transferred to the next block; MIMO block to process the rectified symbol based on the Vertical-Bell Laboratories Layered Space-Time (V-BLAST) criterion. V-BLAS technology is used to attain the system capacity/throughput enhancement, which is expressed in terms of bits/symbol.

The transmitter stages are shown in Figure 7. The transmitted signal through I antennas will be guaranteed to be with the minimum PAPR value, since the resultant unaffected MIMO-OFDM signal will be based on the constant enveloped transformed signal using PWM. As a result, the novelty of this work rises from the way of dealing with the OFDM symbol for such application. This is in addition to the way of how to reduce the CCDF curves values that are guaranteed to remains at their minimum levels.

In the receiver side, the signal modelling will be determined based on the sent and received signals between/ among the transmitter and the object(s), which will help in determining the objects specifications. The issues of determining the objects directions and locations are considered out of scope of this work and will be discussed in another work. Thus, it is focusing on how to overcome the rise problem due to the use of the FFT and its inverse in modelling the OFDM system.

After the transmission through a channel from different transmitting antennas, in the receiver side, the main task of the receiver is to recover the original OFDM signal from the modified one. Accordingly, the used recovering

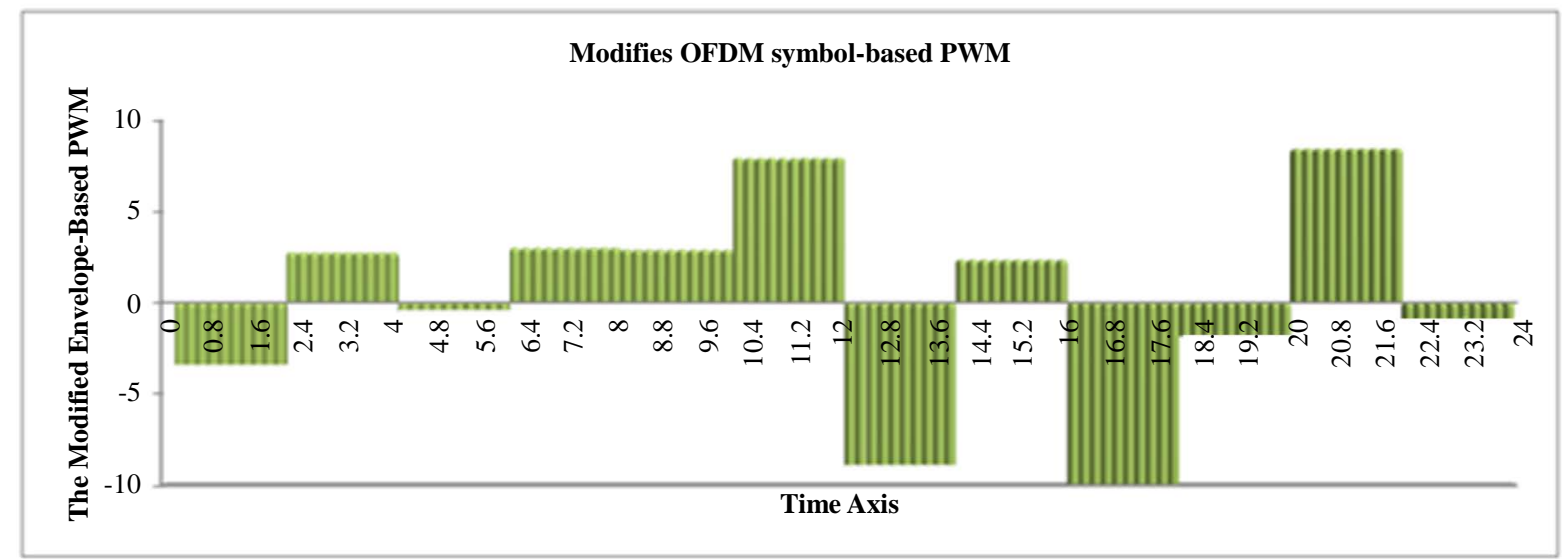

Figure 6. Modified OFDM symbol based PWM.

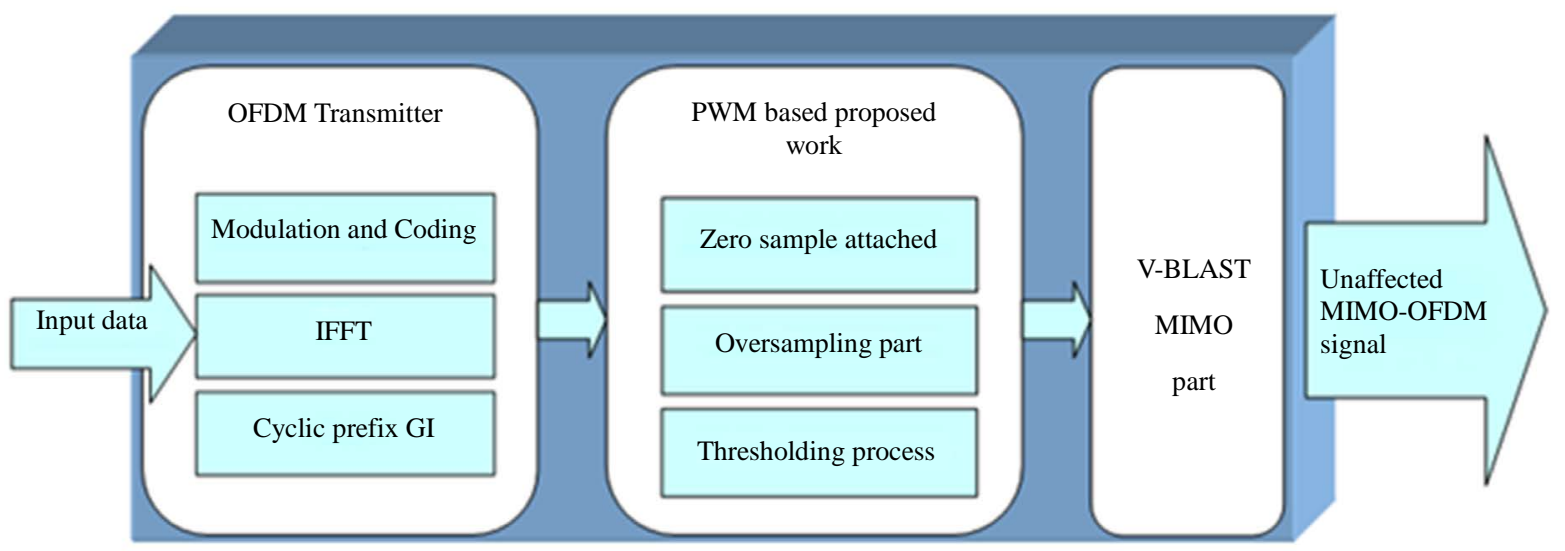

Figure 7. The proposed MIMO-OFDM radar transmitter. 
procedure will be divided into two main stages; firstly, proposing an algorithm to recover the OFDM symbol from the constant enveloped received symbols, and secondly, a signal processing stage based on removing the extra imposed samples. This procedure is clearly shown and described in Figure 8. It contains two parts depicted the proposed processing stages; Figure 8(a) and Figure 8(b) according to stage 1 and stage 2 consequently.

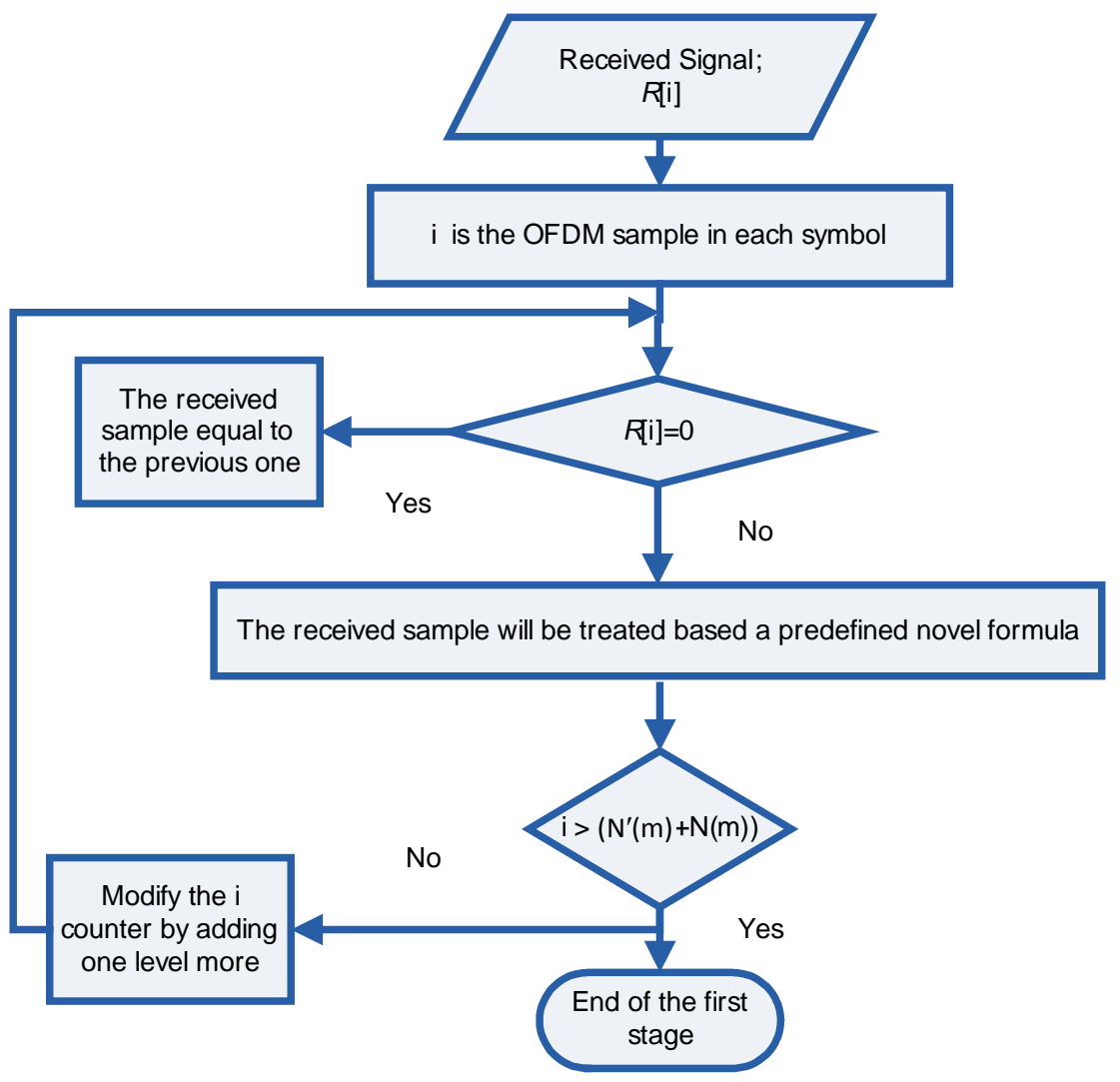

(a)

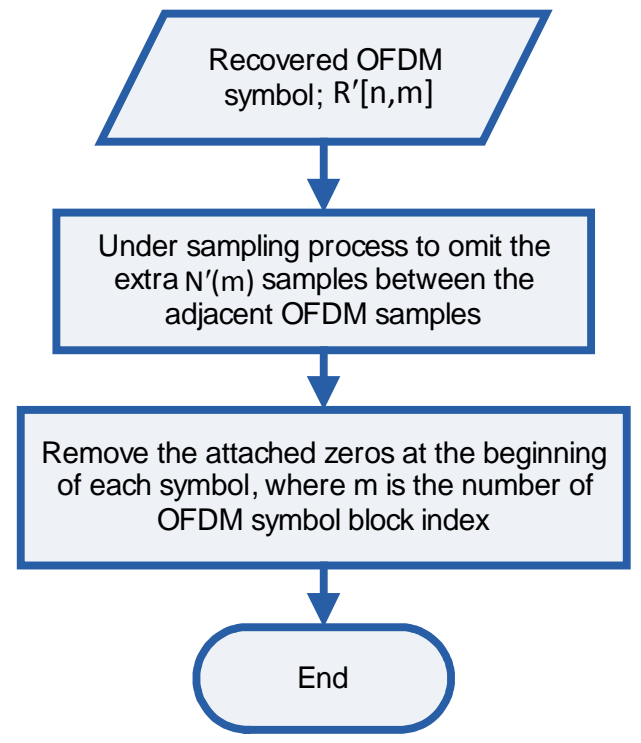

(b)

Figure 8. Reception stage flowchart (a) First stage procedure; (b) Second stage procedure. 
The reproduction process of regenerating OFDM signal from the received is clearly depicted in Figure 8. It is divided into two main parts; the reconversion stage and the de-processing stage; shown in Figure 8(a) and Figure $\mathbf{8}(\mathrm{b})$ respectively.

The given procedure in Figure 8(a) starts with checking the received samples to clarify whether it is the beginning of new symbol or not as shown in (10). Moreover, a novel formula has been proposed to reproduce the generation of the original OFDM symbols based on three different variables; the received sample, the difference in time between a consecutive samples and the previous sample.

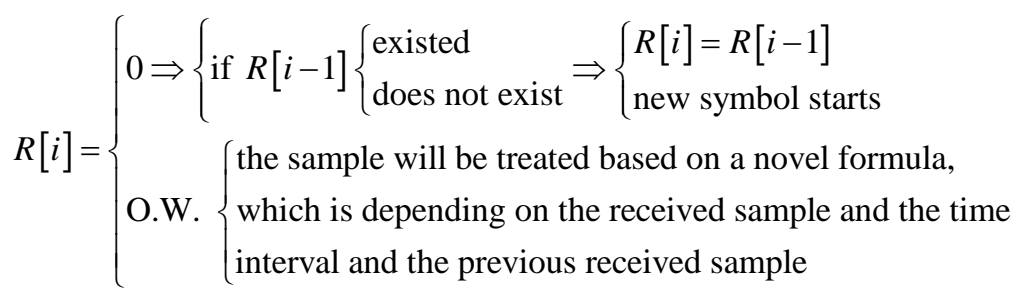

Furthermore, in Figure 8(b) the conversion process has been completed by fulfilling the removal of the extra added samples. The novelty in this work has been shown in how to deal with the OFDM symbol; imposing hard decision criterion based on the PWM instead of sending the original OFDM signals.

The next section describes the results from the proposed work simulation against the conventional techniques; they are based on both of CCDF and SER curves. These two criteria are used to validate the OFDM systems performance, where the lower the values the higher the system performance.

\section{Tested System Performance and Simulated Results}

The proposed MIMO-OFDM radar system that has been described in Figure 7 is used to localise a composite target based on a four-element array. The composite target has been placed far away from the array, which contains three dielectric elements. They are differed in physical dimensions that are given in terms of the carrier frequency but have same dielectric permittivity. Moreover, the used array is assumed to contain isotropic elements with equal spacing. The scope of this work covers the system performance based on tackling the PAPR problem in the MIMO-OFDM radar. This leaves a room to enhance the performance from different point of views for future work.

At this stage, the performed MATLAB simulation has the following specifications:

- Carrier frequency of $3 \mathrm{GHz}$,

- Extra 9 samples have been added between the consecutive samples; $N^{\prime}(\mathrm{m})=9$,

- IFFT length of 1024 point,

- $T_{p}=0.25 \times T_{s}$,

- $\quad$ Carrier spacing = $(1 / 32) \mathrm{GHz}$,

- 64-QAM modulation technique,

- Vertical-Bell Laboratories Layered Space-Time (V-BLAST) MIMO system will be used for the four-element array transmission. This is in order to boost the system performance in terms of bits/symbol.

To imitate a real scenario, the shown proposed work in Figure 7 has been imposed twice; one for the real OFDM symbol part and the other one for the imaginary part. Moreover and in order to test the system's performance, the results have been divided into two parts; the complementary cumulative distribution function (CCDF) part and the sample error rate (SER) part. Figure 9 will depict the value of proposed work SER and how promising the achieved values are, while Figure 10 will clarify the enhancement in combating the effect of the PAPR from the CCDF point of view, which is considered as a performance metric independent of the transmitter amplifier. It is defined by how often the PAPR is higher than a given threshold; $\mathrm{PAPR}_{0}$. It is expressed by:

$$
\operatorname{CCDF}\left(\mathrm{PAPR}_{\mathrm{o}}\right)=\operatorname{Probability}\left\{\mathrm{PAPR}>\mathrm{PAPR}_{\mathrm{o}}\right\}
$$

As depicted in Figure 9, the proposed PWM enhances the recovery process of the original OFDM samples. This work is divided into two experiments; the first one based on using the previous sample in order to predict the received sample, while the other one is based on using the average of all samples within the window. The one that is based on the average gives a better SER under the optimization issue between the SER and the delay 
SER of the received OFDM symbol

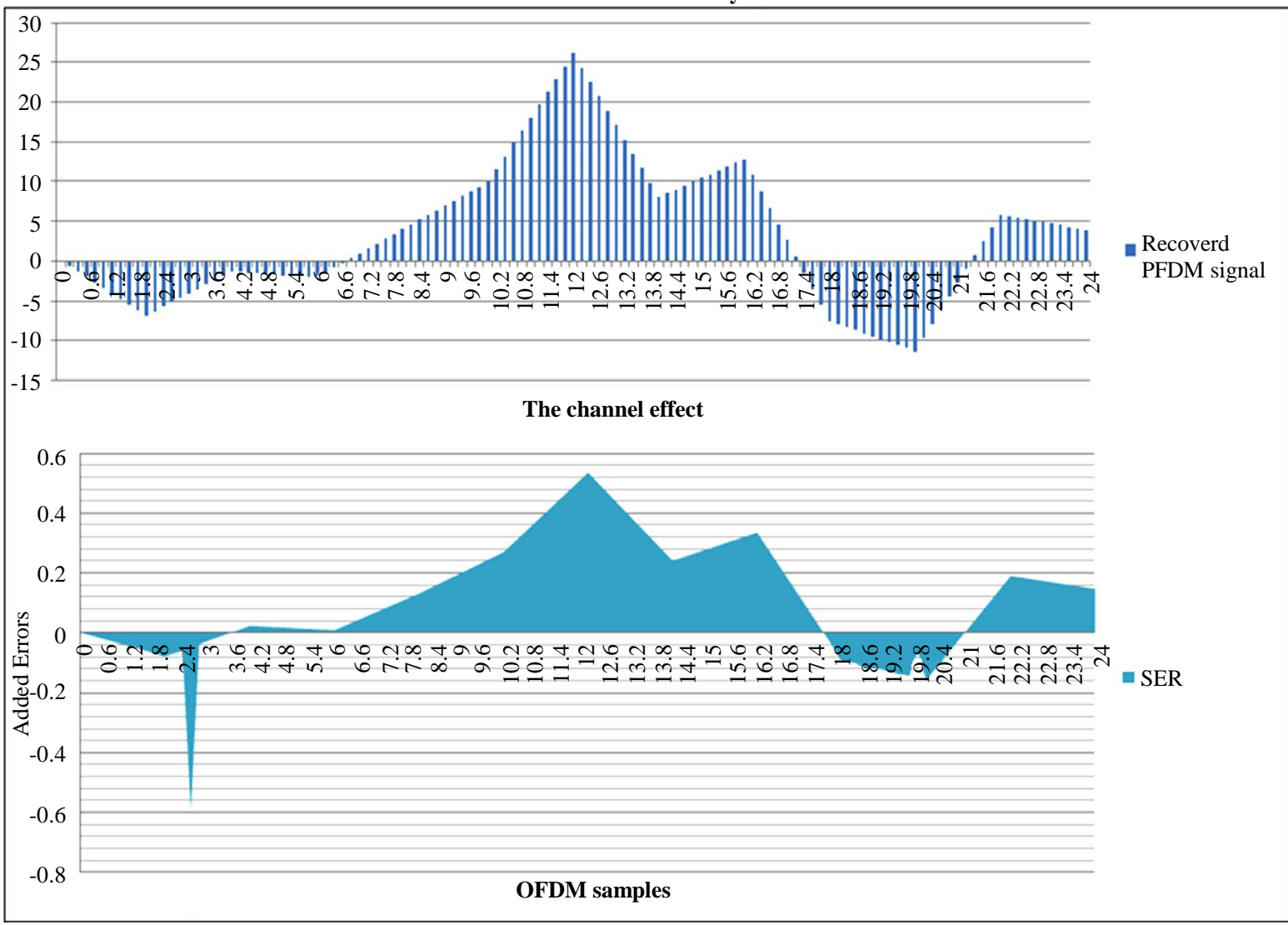

Figure 9. The SER and the recovered OFDM samples.

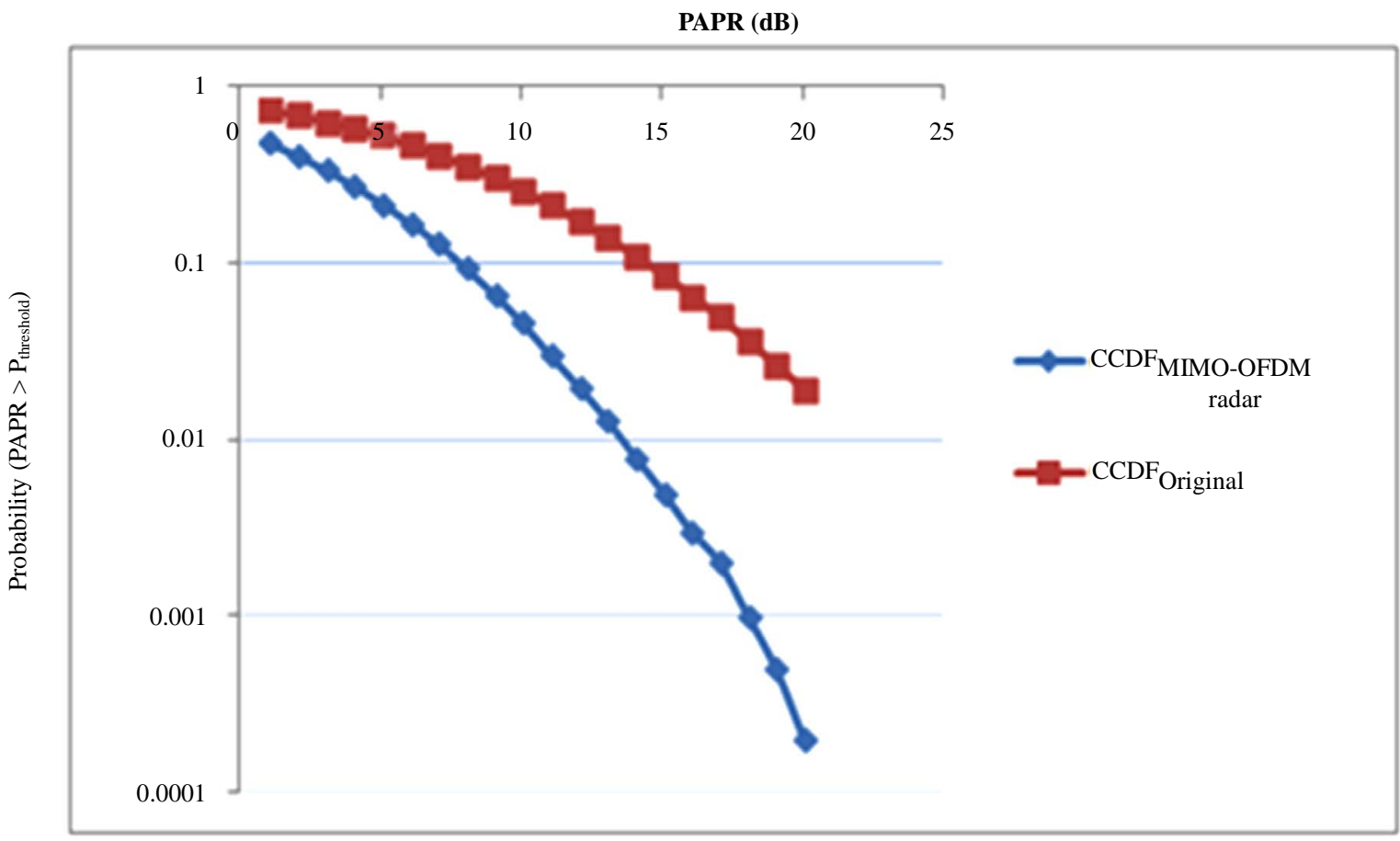

Figure 10. The CCDF results based on 64QAM modulation technique. 
Table 1. The Simulation results of the proposed technique based on PWM to the literature work.

\begin{tabular}{cccccc}
\hline & \multicolumn{2}{c}{ CCDF (2\%) } & \multicolumn{3}{c}{ Additional reduction (\%) } \\
\hline Modulation technique & PAPR without coding (dB) & MIMO-OFDM radar based PWM (dB) & Clipping & SLM & PTS \\
\hline 64 QAM & 20 & 11.5 & 72.1 & 44.5 & 11 \\
\hline
\end{tabular}

time. This modification enhances the SER from $9.3 \times 10^{-4}$ to $8.7 \times 10^{-4}$.

Furthermore, the second part of the systems performance checking is shown in Figure 10 based on the CCDF curves. From the depicted results in Figure 10, the proposed work gives a noticeable improvement in the CCDF curves. Using the PWM as a processing technique to enhance the OFDM signal's envelope reduces the CCDF (20 dB) from $19 \times 10^{-3}$ to $2 \times 10^{-4}$. Furthermore, a comparison has been made between our proposed work and the work that found in the literature. This comparison is shown in Table 1.

The proposed system performance improvement has bean clearly shown in Table 1. It shows that our work has $2 \%$ of the PAPR that is exceeds $11.5 \mathrm{~dB}$, where for the same probability percentage the conventional OFDM systems has a PAPR over $20 \mathrm{~dB}$. Moreover and comparing to the literature, the proposed work has an extra reduction percentage between $11 \%$ and $72 \%$.

MIMO-OFDM radar work is different from the conventional ones in the literature, where this work has built the comparison stage making use of the slope between the two consecutive samples. Additionally, this work enhances the use of the PWM techniques, where the conventional PWM links the comparison performance to the inserted number of extra samples. In the MIMO-OFDM radar, the performance has been improved without overloading the systems with extra samples since the comparison stage has been linked to the slope between the samples.

\section{Conclusions}

This work takes high PAPR effect into consideration when proposing the OFDM technique to the conventional MIMO radar systems. High PAPR values could reduce the system performance especially when using nonlinear devices. A new work has been proposed to overcome this deficiency making use of the conventional PWM with some modifications.

A MATLAB simulation has been conducted to validate the analytical model of the proposed work. It consists of two parts: one to check the sample error rate (SER) after the recovery process, while the other one to describe the probability of the PAPR to exceed a certain threshold. Moreover, a comparison with the literature has been made in order to confirm the expected performance modification.

Under same environmental conditions and system specifications, a SER of $8.7 \times 10^{-4}$ has been achieved compared with the transmission of conventional OFDM signals. This is in addition to enhancing the probability of the PAPR that exceeds $20 \mathrm{~dB}$ from $2.1 \times 10^{-2}$ to $1.7 \times 10^{-4}$. The work validity has been checked based on a comparison with the ones in the literature, such as PTS, SLM or Clipping techniques, the proposed work gives an additional PAPR reduction percentage between $11 \%$ and $72 \%$ over the achieved $11.5 \mathrm{~dB}$ value. As a consequence, the transmission throughput will improve.

\section{References}

[1] Nee, R. and Prasad, R. (2000) OFDM for Wireless Multimedia Communications. Artech House, Norwood.

[2] Umali, E., Toyama, Y. and Yamao, Y. (2008) Power Spectral Analysis of the Envelope Pulse-Width Modulation (EPWM) Transmitter for High Efficiency Amplification of OFDM Signals. IEEE Vehicular Technology Conference (VTC), Singapore, 1261-1265.

[3] 3GPP, Tech. Specif. Group Services and System Aspects Service Requirements for Evolution of the 3GPP System (Rel. 8), 3GPP TS 22.278.

[4] Dahlman, E., et al. (2008) 3G Evolution: HSPA and LTE for Mobile Broadband. 2nd Edition, Academic Press.

[5] Abeta, S. (2010) Toward LTE Commercial Launch and Future Plan for LTE Enhancements (LTE-Advanced). IEEE International Conference on Communication Systems (ICCS) Proceedings, Singapore, 146-150.

[6] Andrews, J., Ghosh, A. and Muhamed, R. (2007) Fundamentals of WiMAX: Understanding Broadband Wireless Networking. Prentice Hall. 
[7] Jiang, T. and Yu, Y. (2008) An Overview: Peak-to-Average Power Ratio Reduction Techniques for OFDM Signals. IEEE Transaction on Broadcasting, 54, 257-268. http://dx.doi.org/10.1109/TBC.2008.915770

[8] Saeed, A., Elbably, M. and Abdelfadeel, G. (2009) Efficient FPGA Implementation of FFT/IFFT Processor. International Journal of Circuits, Systems and Signal Processing, 3, 103-110.

[9] Foschini, G. and Gans, M. (1998) On Limits of Wireless Communications in a Fading Environment When Using Multiple Antennas. Wireless Personal Communications, 6, 311-335. http://dx.doi.org/10.1023/A:1008889222784

[10] Telatar, E. (1995) Capacity of Multi-Antenna Gaussian Channels’. AT \& T Bell Laboratories.

[11] Zelst, V. and Schenk, T. (2004) Implementation of a MIMO OFDM-Based Wireless LAN System. IEEE Transactions on Signal Processing, 52, 483-494. http://dx.doi.org/10.1109/TSP.2003.820989

[12] Bekkemani, I. and Tabrkian, J. (2006) Target Detection and Localization Using MIMO Radars and Sonars. IEEE Transactions on Signal Processing, 54, 3873-3883. http://dx.doi.org/10.1109/TSP.2006.879267

[13] Wang, Y. and Luo, Z. (2011) Optimized Iterative Clipping and Filtering for PAPR Reduction of OFDM Signals. IEEE Transactions on Communications, 59, 33-37. http://dx.doi.org/10.1109/TCOMM.2010.102910.090040

[14] Chen, J.C. (2010) Partial Transmit Sequence for PAPR Reduction of OFDM Signals with Stochastic Optimization Techniques. IEEE Transactions on Consumer Electronics, 56, 1229-1234. http://dx.doi.org/10.1109/TCE.2010.5606251

[15] Sohn, I. (2014) A Low Complexity PAPR Reduction Scheme for OFDM Systems via Neural Networks. IEEE Communications Letters, 18, 225-228. http://dx.doi.org/10.1109/LCOMM.2013.123113.131888

[16] Wang, F.P., Kimball, D., Popp, J., Yang, A., Lie, D., Asbeck, P. and Larson, L. (2005) Wideband Envelope Elimination and Restoration Power Amplifier with High Efficiency Wideband Envelope Amplifier for WLAN 802.11g Applications. IEEE Microwave Symposium Digest, Long Beach, 12-17 June 2005, 645-648.

[17] Vasca, F. and Lannelli, L. (2012) Dynamics and Control of Switched Electronic Systems: Advanced Perspectives for Modelling, Simulation and Control of Power Converters. Springer Publisher, Berlin.

[18] Koyuncu, M., van den Bos, C. and Serdijn, W. (2000) A PWM Modulator for Wireless Infrared Communication. Proceedings of the ProRISC/IEEE Workshop on Semiconductors, Circuits, Systems and Signal Processing, Veldhoven, 30 November-1 December 2000, 351-353.

[19] Ochiai, H. and Imai, H. (2001) On the Distribution of the Peak-to-Average Power Ratio in OFDM Signals. IEEE Transactions on Communications, 49, 282-289. http://dx.doi.org/10.1109/26.905885

[20] Wei, S., Goeckel, D. and Kelly, P. (2010) Convergence of the Complex Envelope of Bandlimited OFDM Signals. IEEE Transactions on Information Theory, 56, 4893-4904. http://dx.doi.org/10.1109/TIT.2010.2059550

[21] Lin, H. and Siohan, P. (2008) OFDM/OQAM with Hermitian Symmetry: Design and Performance for Baseband Communication. IEEE International Conference on Communications (ICC’ 08), Beijing, 19-23 May 2008, 652-656. 
Scientific Research Publishing (SCIRP) is one of the largest Open Access journal publishers. It is currently publishing more than 200 open access, online, peer-reviewed journals covering a wide range of academic disciplines. SCIRP serves the worldwide academic communities and contributes to the progress and application of science with its publication.

Other selected journals from SCIRP are listed as below. Submit your manuscript to us via either submit@scirp.org or Online Submission Portal.
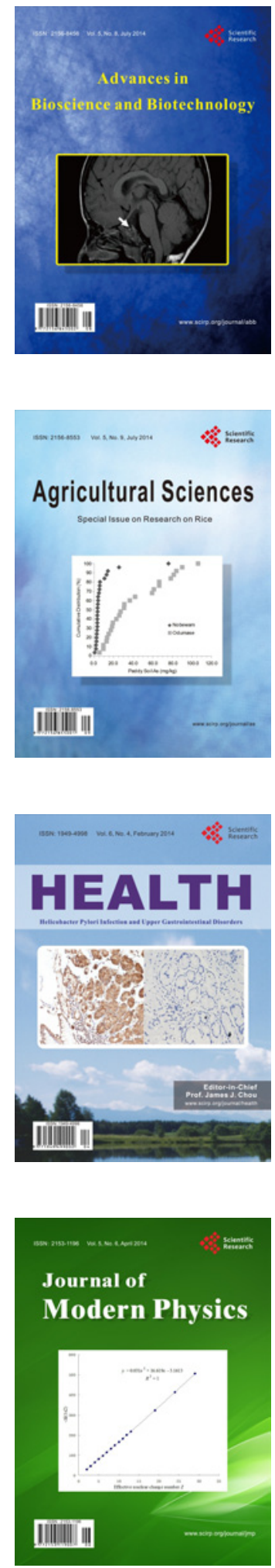
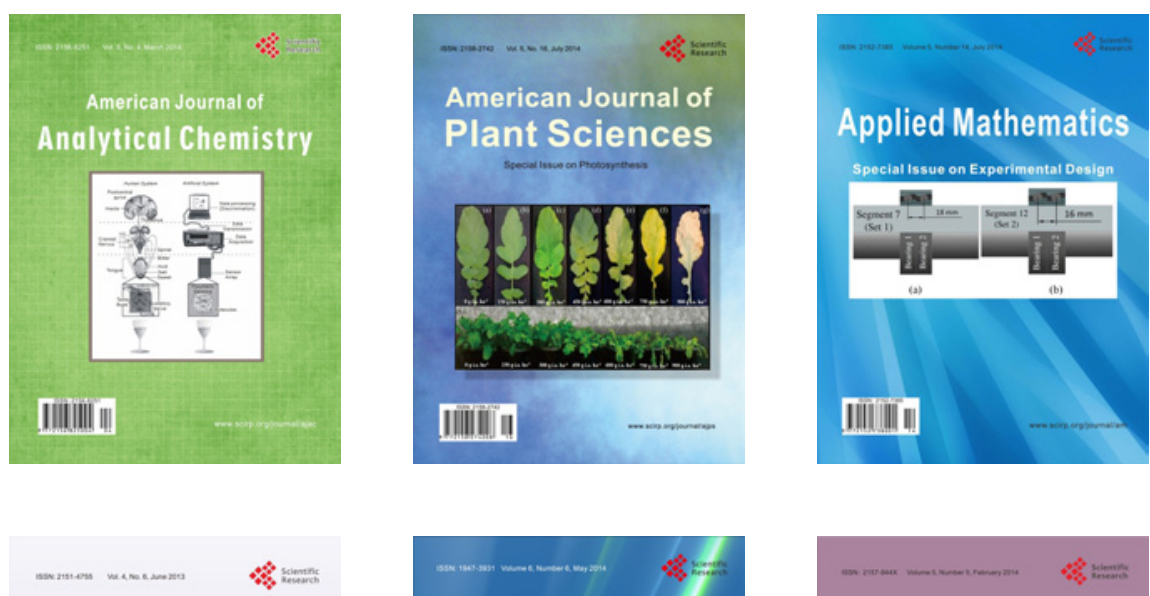

Creative Education
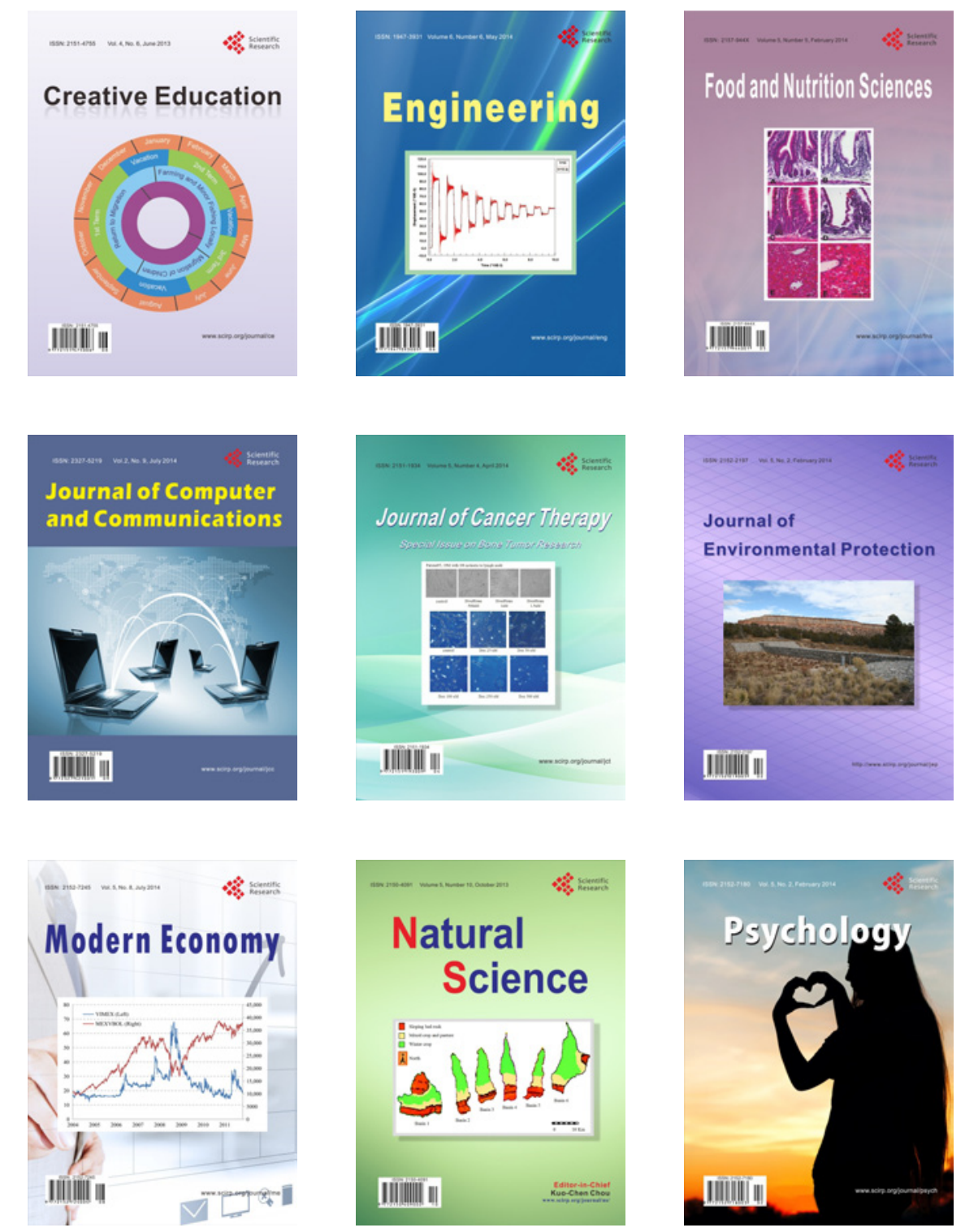